\title{
tic\&société
}

Vol. 10, N 2-3 | 2ème semestre 2016 - 1er semestre 2017

Spécial varia

TIC et démocratie locale. Considérations empiriques issues de la pratique du budget participatif dans des communes camerounaises

\section{Calvin Minfegue}

\section{OpenEdition \\ Journals}

Édition électronique

URL : http://journals.openedition.org/ticetsociete/2100

DOI : 10.4000/ticetsociete. 2100

Éditeur

Association ARTIC

Référence électronique

Calvin Minfegue, "TIC et démocratie locale. Considérations empiriques issues de la pratique du

budget participatif dans des communes camerounaises », tic\&société [En ligne], Vol. 10, N² 2-3 | 2ème semestre 2016 - 1er semestre 2017, mis en ligne le 30 avril 2017, consulté le 01 mai 2019. URL :

http://journals.openedition.org/ticetsociete/2100; DOI : 10.4000/ticetsociete.2100 
tic\&seciété - 10(2-3), 2016-2017

TIC et démocratie locale. Considérations empiriques issues de la pratique du budget participatif dans des communes camerounaises

\author{
Calvin MINFEGUE
}

Calvin Minfegue est doctorant en sciences sociales à l'Université catholique d'Afrique centrale (UCAC) à Yaoundé (Cameroun) après avoir suivi des études en géographie et en sciences politiques. Il est chercheur associé à l'Institut des Politiques et Initiative sociales (IPIS) au Cameroun et à Modus Operandi en France. Ses recherches portent entre autres sur les dispositifs participatifs et leurs mutations en contexte local (avec un accent sur leur association aux TIC), les dynamiques (trans)frontalières en Afrique centrale et la problématique des conflits. Il a participé à des projets de recherche dans ces différents champs.

minassc@yahoo.fr 


\section{Calvin MINFEGUE}

\section{TIC et démocratie locale. Considérations empiriques issues de la pratique du budget participatif dans des communes camerounaises}

Résumé : Dans le sillage des travaux sur les usages des TIC, la présente réflexion s'intéresse à l'emploi de ces outils dans les dynamiques politiques et démocratiques à l'échelon local en contexte camerounais. La pratique du budget participatif étant mobilisée comme champ d'observation, l'article se décline en trois grands points. Il examine d'abord les conditions originelles de la mobilisation des TIC dans la pratique du budget participatif dans les communes ciblées et, ensuite, les usages associés aux TIC dans ces pratiques sont explicités. Enfin, il est question de discuter des données présentées dans la perspective plus ample des débats théoriques sur l'e-démocratie.

Mots-clés: TIC, budget participatif, usages, dynamique politique, participation citoyenne.

Abstract: Following various works on uses of ICTs, this paper focuses on their mobilization in local political dynamics in Cameroon. We observed participatory budgeting practices. This analysis first describes the initial introduction of ICTs in participatory budgeting and then details the various uses of ICT in this process. Finally, It discusses these results in the global framework of debates on e-democracy.

Keywords: ICT, participatory budgeting, uses, politics, citizen participation.

Resumen: En el ámbito de los trabajos sobre los usos de las TIC, este texto analiza el empleo de las TIC en las dinámicas políticas y democráticas a nivel local en el contexto del Camerún. Apoyándose sobre el caso del presupuesto participativo, el presente artículo se organiza 
TIC et démocratie locale. Considérations empiriques issues de la pratique du budget participatif dans des communes camerounaises

en tres partes. En primer lugar, examina las condiciones originales de la introducción de las TIC en el presupuesto participativo en determinados municipios. En segundo lugar se consideran los usos sociales asociados a las TIC. Finalmente, se discuten los datos obtenidos en una perspectiva más amplia, en el marco de los debates teóricos sobre la e-democracia.

Palabras clave: TIC, presupuesto participativo, usos, dinámica política, participación ciudadana. 


\section{Calvin MINFEGUE}

Des usages multiformes qui sont faits des techniques de l'information et de la communication (TIC) ${ }^{1}$ en Afrique, certains, par exemple dans le cadre de processus politiques, apparaissent peu explorés aujourd'hui, notamment dans le cas de l'Afrique centrale. Même si le couple TIC et politique semble fécond au premier chef, dans cette partie du continent, il souffre d'un approfondissement insuffisant sur le plan analytique. L'analyse des usages par les politiques et pour la politique est très popularisée, si on se réfère à l'intense exploitation qui est faite des réseaux sociaux (Savah et Galabov, 2012 ; Zakhour, 2011). Pourtant, d'autres formes d'utilisation existent et demeurent peu examinées. Le Cameroun n'échappe pas à cette abondance. On y repère des expériences de ce type, tant au niveau du système politique central qu'au niveau local. Justement au niveau infranational, des municipalités mettant en œuvre le budget participatif (BP) s'avèrent être les principaux laboratoires d'expérimentation associant les TIC aux enjeux sociopolitiques. Ces initiatives, qui se sont multipliées au cours des cinq dernières années, doivent être analysées en mettant l'accent sur leur portée.

Le présent article se propose d'examiner, à partir des cas de communes camerounaises mettant en œuvre le BP, l'usage fait des TIC dans les processus politiques au niveau local. Il s'agira, après avoir posé des préalables méthodologiques et contextuels, d'examiner les conditions originelles de la mobilisation des TIC dans la mise en œuvre du BP, d'en expliciter les usages et les primo effets et, enfin, de discuter de ces résultats dans la perspective des constructions théoriques, plus amples, liées notamment à la démocratie dite « électronique », l'e-démocratie.

\footnotetext{
${ }^{1}$ Nous nous situons ici dans la perspective d'Éric George (2014) qui préfère parler de technique plutôt que de technologie. II rappelle à ce propos que la technologie réfère étymologiquement à un discours (logos) sur la technique.
} 
TIC et démocratie locale. Considérations empiriques issues de la pratique du budget participatif dans des communes camerounaises

\section{Préalables méthodologiques et contextuels}

\subsection{Les prémisses}

Avoir la prétention de publier un article comprenant un ancrage aussi empirique dans la revue tic\&société pourrait relever d'un anachronisme factuel quand on connaît la portée critique des publications qui y paraissent. Pour notre défense, permettons-nous de rappeler simplement que les perspectives empiriques renforcent ou complètent de nombreux travaux à orientation critique. Le choix a été fait d'interroger, ici, les apports des TIC à la dynamique démocratique (une dynamique d'abord sociale), notamment dans leur capacité à donner des contours nouveaux aux modalités de participation citoyenne, un critère démocratique (Dahl, 1998), en contexte africain. L'accent sur le local est, ici, choisi, car il permet, dans un système démocratique, de saisir plus subtilement les effets des décisions sur les citoyens (Pailliart, 1995 ; Peixoto, 2008). À partir de l'échelon municipal, nous portons une appréciation sur la mobilisation des TIC dans la dynamique démocratique locale. Sur ce point, c'est l'expérience du BP, introduite au Cameroun en 2003, qui est valorisée. Il existe une littérature abondante sur cette pratique née à la fin des années 1980 au Brésil ; nous n'y reviendrons pas. Rappelons simplement que le BP est « un mécanisme [...] par lequel les populations décident de l'affectation de tout ou partie des ressources publiques ou sont associés aux décisions relatives à cette affectation » (Genro, Desouza, 1998, cité par Cabannes, 2006, p. 128).

Examiner la mobilisation des TIC dans le BP nous conduit à analyser leur place dans les processus politiques, particulièrement dans le modèle démocratique camerounais. Depuis les années 1970, des auteurs ont en effet vu dans l'avènement des TIC une opportunité de renforcer la démocratie représentative (Laudon, 1977) ou de l'orienter radicalement vers un modèle plus délibératif, plus direct (Barber, 1984). Les TIC ont toujours été perçues comme un facteur de transformation du modèle démocratique. Pour sa part, Dahl (1998) présente les axes par lesquels les TIC peuvent être utiles à la démocratie. Il estime que l'un des enjeux les plus urgents des démocraties est "d'améliorer les capacités du citoyen afin de lui permettre de s'engager avec intelligence dans la vie politique» 
(p. 179). Les TIC, de son point de vue, sont capables de renforcer, d'améliorer et de rafraîchir les options déjà existantes de formation des citoyens. Ces outils étaient déjà considérés comme possédant un « caractère politique » du fait des "possibilités » qu'ils offraient « de favoriser la démocratie locale » (Pailliart, 1995, p. 69). Au croisement de la démocratie et des TIC, l'e-démocratie peut être appréhendée comme cette forme de démocratie sous-tendue par la possibilité de mobiliser les TIC dans les processus politiques, notamment sur le plan de l'information, de la délibération/discussion et de la prise de décisions (Liden, 2012), et ceci dans un environnement où les libertés et les droits civiques sont garantis. Toutefois, la portée de l'edémocratie est remise en question : permet-elle d'opérer des sauts qualitatifs suffisamment pertinents comparativement à la pratique démocratique ne mobilisant pas les TIC? Renforce-t-elle la participation? Redéfinit-elle les rapports entre gouvernants et gouvernés dans une logique de démocratie "continue » (Rodotà, 1999 ; Rousseau, 1995) ? Ces interrogations seront éprouvées ici, dans le contexte de la mise en œuvre du BP. Les résultats feront l'objet d'une discussion critique. Ainsi, ce travail s'inscrit dans le sillage des réflexions sur l'apport des TIC dans les dynamiques politiques et démocratiques.

\subsection{Le dispositif méthodologique}

Bien qu'implicitement assumées, les réflexions développées ici, en lien avec l'usage des TIC, ont pour finalité d'analyser la fonctionnalité des TIC dans le cadre du BP considéré comme étant une « structure » au sein de laquelle des acteurs agissent. Nous souhaitons aussi avoir une meilleure compréhension de l'utilité associée aux TIC en y singularisant leurs divers emplois. Dans le cadre de cette réflexion, le sigle TIC réfère explicitement à trois outils : une plateforme web-SMS, qui valorise les possibilités d'usage des SMS ; un centre d'appel dit "citoyen", du fait de son orientation vers des enjeux davantage civiques qu'économiques; et, enfin, le réseau socionumérique Facebook.

Les données présentées ont été collectées à travers trois techniques. Une revue de littérature a permis d'examiner des travaux scientifiques sur le sujet et la littérature institutionnelle propre aux 
TIC et démocratie locale. Considérations empiriques issues de la pratique du budget participatif dans des communes camerounaises

expériences décrites ici. Des données issues d'entretiens individuels (semi-structurés) ont été obtenues auprès d'une vingtaine d'acteurs aux profils variés (élus locaux, leaders communautaires, acteurs de la société civile) entre 2012 et 2015. Une observation directe a complété ce dispositif. Elle a permis de suivre des activités du BP, en l'occurrence les fora (forums) de quartiers, les réunions de sensibilisation et la visualisation de l'exploitation de la plateforme web-SMS et du centre d'appel.

\subsection{Les terrains d'observation}

Les données exploitées proviennent de quatre communes camerounaises mettant en œuvre le BP et ayant mobilisé les TIC dans ce processus. Il s'agit des communes d'Edzendouan, des arrondissements de Yaoundé 2 et de Yaoundé 6, dans la région du centre, et de celle de l'arrondissement de Douala 3, dans la région du Littoral. Si les trois dernières communes sont des collectivités urbaines, Edzendouan est une municipalité rurale. Yaoundé 2 et Yaoundé 6 ont implanté le BP depuis 2009, Edzendouan depuis 20032004. Après une interruption en 2007, cette dernière municipalité a renoué avec cette pratique en 2012. Douala 3 a expérimenté le processus pour la première fois en 2012. Sur le plan démographique, Yaoundé 2 et Yaoundé 6 comptaient respectivement environ 300000 et 280000 habitants en 2012. Douala 3 abritait une population estimée à 670000 âmes en 2013, tandis qu'Edzendouan en comptait, selon les sources administratives, environ 6000 en 2011.

Concernant l'équipement en TIC, l'Agence de Régulation des Télécommunications du Cameroun (ART) indiquait que le pays comptait 17,46 millions d'abonnés téléphoniques (téléphonies mobile et fixe) en service à la fin de l'année 2014, soit un taux de pénétration national de $81,49 \%$ (ART, 2016). Au cours de la même année, le nombre d'abonnés à internet s'élevait à "plus» de 5 millions, toutes options confondues (Wimax, 3G, ADSL, etc.). Sur le plan local, une enquête de l'Institut National de la Statistique (INS) conduite en 2011 relevait que le pourcentage de ménages possédant au moins un téléphone portable était d'environ $95 \%$ dans les villes de Yaoundé et Douala. Ce taux était de l'ordre de $46 \%$ en moyenne en zone rurale (INS, 2012). 


\section{Aux origines de l'association TIC-BP au Cameroun}

L'introduction des TIC dans la mise en œuvre du BP au Cameroun remonte à la fin de l'année 2011. Cet usage des TIC, bien que novateur au niveau local, existait déjà dans des expériences d'amélioration des services publics (Gouo, 2008). Localement, des initiatives étaient identifiables au niveau municipal depuis 2010, mais semblaient très fragiles dans leur capacité à tirer parti du potentiel des TIC. L'exploitation de ces outils, dans le contexte des communes mettant en œuvre le BP, a reposé sur une combinaison subtile de facteurs exogènes et d'une trajectoire d'appropriation interne par la société civile.

\subsection{Le poids des motivations et facteurs exogènes}

Dans le cadre de ses actions, l'Institut de la Banque mondiale a soutenu de nombreux gouvernements dans la mise en œuvre de programmes visant l'utilisation des TIC dans les processus démocratiques. Il existe une littérature institutionnelle intéressante sur la capitalisation de ces expériences (Heller, Rao, 2015 ; World Bank, 2011).

En Afrique centrale, l'un des premiers programmes de ce type fut expérimenté en 2009 en République Démocratique du Congo (RDC), dans la province du Sud-Kivu. Ce Programme, dénommé «ICT 4 GOV» («ICT for Governance», ou «TIC pour la gouvernance»), s'inscrivait dans le cadre global de soutien à la décentralisation dans ce pays. L'objectif était de faciliter ce processus à travers un renforcement de la capacité de participation des acteurs (locaux) au BP par l'usage des TIC. La finalité consistait alors à améliorer la gouvernance et la qualité des services publics rendus aux citoyens dans cette province, tout en ouvrant la voie à des réformes plus structurelles. L'introduction du BP au Sud-Kivu avait été facilitée par un travail collaboratif (partage d'expériences) entre des organisations de la société civile de la RDC et du Cameroun (en l'occurrence Actions solidaires de soutien aux organisations et d'appui aux Libertés [ASSOAL]). L'évaluation préliminaire de cette expérience a montré que les TIC, en l'occurrence les applications mobiles, étaient un atout pour ce processus (World Bank Institute, 2012). Elles permettaient de renforcer le niveau de confiance des 
TIC et démocratie locale. Considérations empiriques issues de la pratique du budget participatif dans des communes camerounaises

acteurs à travers des possibilités accrues de communication et de participation. Toutefois, à propos de la question des effets des TIC sur la mobilisation et sur l'implication dans la prise de décisions dans le cadre global du BP, de nombreux facteurs liés à la conception même du projet, mais aussi à la topographie politique du territoire ciblé, n'ont pas permis de parvenir à des conclusions suffisamment rigoureuses. À titre illustratif, le suivi des indicateurs du projet ne s'est pas inscrit dans un cadre coopératif et optimal avec les institutions locales. Une telle orientation aurait permis d'anticiper sur ses résultats. Les lourdeurs liées à la décentralisation ont parfois limité la marge d'action des autorités locales et, partant, leur engagement constant dans le projet. Cette expérience a néanmoins permis de poser les bases des futures initiatives de cette nature.

Un programme similaire a été développé au Cameroun. Le pays avait l'avantage de justifier d'un passif intéressant de pratique du BP depuis 2003. L'antériorité des relations entre certaines ONG locales et des partenaires internationaux comme l'Union européenne y donnait des garanties de déploiement. ASSOAL a donc été choisie comme relais local sur la base, principalement, de son statut d'organisation pionnière en matière d'accompagnement des expériences de $\mathrm{BP}$, mais aussi de rapports établis en $\mathrm{RDC}$, où elle avait accompagné des acteurs locaux dans l'expérimentation du processus. À la fin de l'année 2011, la décision fut prise d'expérimenter cette approche de BP reposant sur les TIC dans deux communes (bien que quatre ont initialement été ciblées), à savoir Yaoundé 2 et Yaoundé 6. Ce projet expérimental baptisé «ICT 4 PB » («ICT for Participatory Budgeting», soit «TIC pour le BP»), a démarré en mars 2012.

Sur un plan technique, dans la continuité de l'expérience du SudKivu, le projet a principalement mobilisé les Short Message Services (SMS), service lié à la téléphonie mobile. À travers une plateforme web-SMS, système qui intègre le SMS et les possibilités de flexibilité, d'interaction propres au réseau internet, les messages sont, en théorie, utilisés pour faciliter l'information et la mobilisation des citoyens, renforcer la communication municipale et participer à l'implication citoyenne dans la prise des décisions. En vue de tester la faisabilité de cette action, les communes ciblées étaient mobilisées à 


\section{Calvin MINFEGUE}

des étapes différentes du processus : la municipalité de Yaoundé 2 l'était pour le second cycle du BP, celui de l'exécution des projets retenus dans le cadre du dispositif, et Yaoundé 6, pour le premier cycle, soit la priorisation des initiatives.

Ce projet apparait comme l'une des initiatives ayant permis une utilisation intense et explicite des TIC dans des dynamiques sociopolitiques au niveau local. Le fait qu'il ait été co-porté par l'Institut de la Banque mondiale lui donne une teinte partiellement extravertie. Autrement dit, il a été impulsé de l'« extérieur » (Banque mondiale) plutôt que de l'intérieur (par les autorités locales ou la société civile nationale).

\subsection{Une appropriation interne portée par la société civile}

Bien qu'en partie impulsée par des agents externes, l'association entre TIC et BP a fait l'objet d'une appropriation endogène portée principalement par ASSOAL. Cette appropriation s'est effectuée en deux temps d'après les données obtenues auprès des responsables de cette structure.

Le premier temps a porté sur la "responsabilisation d'ASSOAL dans la mise en œuvre du projet "ICT $4 \mathrm{~PB}^{2}$ ». Il reposait sur la dimension collaborative du projet qui précisait les actions respectives des principaux partenaires engagés, à savoir l'Institut de la Banque mondiale et ASSOAL. Ce dernier était responsable de la mise en œuvre du projet sur le plan opérationnel. À cet égard, même les activités conduites par l'Institut de la Banque mondiale reposaient sur un travail concerté avec ASSOAL, s'inscrivant ainsi dans une logique de transfert d'expertise. Cette responsabilisation est à la base de la maîtrise progressive, par cette structure, de l'usage des TIC dans les processus participatifs. Cette organisation s'est néanmoins inscrite dans une dynamique collaborative, permettant une appropriation qui s'est élargie par la suite à un nombre plus important d'organisations.

2 Entretien réalisé le 11 mars 2014 à Yaoundé avec le responsable technique de la mise en œuvre de l'action, par ailleurs en charge de l'axe « Gouvernance » à ASSOAL. 
TIC et démocratie locale. Considérations empiriques issues de la pratique du budget participatif dans des communes camerounaises

Le second temps de cette appropriation a consisté à intéresser un plus grand nombre d'organisations de la société civile (OSC), ceci dans le but de garantir une meilleure dissémination des pratiques d'usage des TIC dans les processus de gouvernance locale. Cette suscitation d'intérêt s'est faite par deux actions: d'une part, la mobilisation des réseaux d'organisations de la société civile, comme le Réseau National des Habitants du Cameroun (RNHC), l'Alliance Camerounaise pour le BP et la Finance Locale (ACBPFL); d'autre part, une présence plus importante aux fora, conférences et évènements publics sur la thématique de la participation.

La première action a permis de s'appuyer sur des organisations travaillant sur la sensibilisation et la dissémination du BP en vue de les impliquer dans l'expérimentation de cet usage politique et local des TIC. Aussi, des organisations membres du RNHC et de l'ACBPFL se sont fortement impliquées dans les activités du projet expérimental, surtout dans sa dimension «mobilisation communautaire » (information, sensibilisation, etc.), à l'instar de l'Association des jeunes d'Edzoa Mballa (AJEM) et du Centre de ressources pour le développement local (CRDL). Le choix de ces réseaux d'organisations n'est pas totalement neutre. Il s'agit, en effet, de structures au sein desquelles ASSOAL joue un rôle majeur en matière d'animation. Au vu des informations recueillies auprès de ses responsables, ASSOAL a assuré, entre 2010 et 2014, le secrétariat technique du RNHC et le secrétariat permanent de l'ACBPFL.

La seconde action a permis de sensibiliser les citoyens au sujet des bienfaits de la mobilisation des TIC dans les processus participatifs autant que dans l'amélioration de la gouvernance publique. Les TIC sont considérées par les OSC comme étant un atout pour renforcer la transparence et la participation aux affaires publiques, tant aux niveaux local que national. Elles contribueraient à créer un cercle vertueux de redevabilité permettant de redéfinir la nature des relations entre gouvernants et gouvernés. Les TIC permettraient de combler le vide existant entre les initiatives gouvernementales et les besoins des citoyens en lien avec le BP ; elles auraient donc une utilité sur le plan du renforcement des liens entre élus et citoyens. Elles agiraient sur la capacité des premiers à répondre aux sollicitations des seconds et à la propension des citoyens à transmettre des 
demandes aux élus (Gigler, Bailur et Anand, 2014). Ces aspects apparaissent centraux dans un processus de BP.

L'adoption des TIC dans les municipalités camerounaises, comme levier du BP, semble avoir reposé de façon importante sur des facteurs exogènes. Des déterminants endogènes, tels que les volontés locales d'amélioration des modes de gouvernance, quoique réels, semblent avoir été initialement secondaires. Ces capitaux internes se sont traduits par une dynamique stratifiée d'appropriation, qui s'est fondamentalement inscrite dans une option de pérennisation de l'action expérimentale «ICT 4 PB ».

\section{Des usages ambivalents des TIC dans les expériences politiques locales : le «prince » garde la main}

Pour les communes ciblées ici, les usages des TIC dans le BP semblent s'organiser autour d'emplois qui s'avèrent soit manifestes soit détournés, selon les cas.

\subsection{Des usages manifestes calqués sur le modèle classique de "bonne gouvernance "}

Par usage manifeste, nous faisons référence au recours explicitement reconnu (et recherché) aux TIC par les acteurs sociaux. Il s'agit, ici, de la mise en exergue de la fonction systématiquement associée aux TIC dans le processus du BP. Dans le contexte des communes ciblées, ces usages s'adaptent aux axes-clés du modèle (commun) de "bonne gouvernance ", à savoir l'accès à l'information associée à la participation des acteurs aux processus décisionnels (PNUD, 2000). Ils ont spécifiquement concerné le renforcement des mécanismes d'information autour du BP, de la gouvernance locale et de la génération de nouvelles opportunités d'implication directe des citoyens aux processus décisionnels.

3.1.1. Une optimisation des mécanismes locaux d'information 
TIC et démocratie locale. Considérations empiriques issues de la pratique du budget participatif dans des communes camerounaises

L'accès à l'information a toujours constitué un enjeu fondamental des initiatives d'amélioration de la gouvernance. Cette question devient capitale sur un plan local, dans la mesure où l'institution municipale a une exigence de proximité vis-à-vis des citoyens. Cette proximité a un positionnement communicationnel tout à fait explicite: faciliter l'accès des citoyens aux informations qui concernent les « affaires de la cité ». Une telle démarche correspond à la fois à « une stratégie de publicisation » de l'action publique locale et à une rupture avec un mode de gestion " notabiliaire » aujourd'hui dépassé (Pailliart, 1995, p. 70). Cela suppose de disposer de canaux performants qui soient à même de délivrer les bons messages au plus grand nombre. Les TIC semblent à cet effet présenter deux principaux avantages: leur potentiel à toucher simultanément de nombreux citoyens et leur caractère peu onéreux.

À Douala 3, Edzendouan et Yaoundé 2, les outils mobilisés entre 2012 et 2015 ont été les SMS et les plateformes web des réseaux socionumériques et d'autres sites web. Ces outils ont permis de renforcer les mécanismes d'information autour du processus du BP et d'autres aspects-clés de la gouvernance locale. Il s'agit bien d'un renforcement de la communication, dans la mesure où ces outils ne se substituent pas aux dispositifs traditionnels (recours aux médias de masse, comme les chaînes locales de télévisions et de radio, ou à l'affichage physique sous les formats d'affiches ou de dépliants informatifs). Deux aspects peuvent être mis en exergue : la capacité des TIC à diversifier et à personnaliser des messages spécifiques (types de messages, accent sur la diversité) et leur portée, soit la capacité à toucher directement, instantanément et simultanément un nombre important de personnes comparativement aux mécanismes traditionnels.

Le recours aux TIC, notamment aux SMS, a permis de diversifier les contenus des informations à destination des citoyens. Avant 2012, les messages provenant de la municipalité, et portant sur le BP, concernaient la tenue de réunions communautaires (fora de quartier, de délégués et assemblées communales). Les canaux mobilisés étaient essentiellement l'affichage des informations dans des lieux de grande fréquentation (marchés, carrefours, chefferies locales, etc.), l'envoi de courriers ciblés aux leaders communautaires (chefs de 


\section{Calvin MINFEGUE}

quartiers et de blocs ${ }^{3}$ ) et, accessoirement, les communications radiophonique et télévisée.

Dans le cas de la commune d'Edzendouan, le recours aux relais communautaires (acteurs locaux chargés de diffuser les informations) était le canal le plus utilisé. Dès 2013, les TIC ont modifié la trame et les contenus des messages élargis à l'information sur les budgets et au répertoire des projets mis en œuvre dans les territoires communaux. Il faudrait noter que l'envoi de SMS permet de cibler directement des citoyens avec la possibilité de personnaliser les contenus comme nous l'avons relevé. À titre d'exemple, les citoyens ayant accepté de recevoir ces informations à Douala 3 et Yaoundé 2 étaient informés de la date et du lieu de tenue d'une activité communautaire liée au BP qui concernait leur quartier. Dans un tel contexte, le risque de déperdition peut apparaître inférieur (réception et stockage du message sur un terminal mobile personnel) à celui lié à un communiqué radiophonique, parfois ponctuel, et dont on est pas certain qu'il sera suivi par les acteurs ciblés.

Les potentialités de ces outils invitent à croire qu'ils peuvent avoir un impact important. Celui-ci peut toutefois largement varier en fonction d'autres éléments de contexte. De plus, il est difficile de mesurer empiriquement l'impact des TIC qui sont combinées avec différents éléments plus traditionnels. Le seul indicateur en la matière pour les acteurs repose sur le résultat obtenu en matière de mobilisation. Un des animateurs du projet à ASSOAL a fait remarquer :

Nous avons obtenu, entre 2012 et 2015, des meilleures mobilisations des populations dans les communes où nous travaillions en utilisant à la fois les TIC et les médias traditionnels. La participation était plus importante que

\footnotetext{
${ }^{3}$ Dans l'architecture urbaine camerounaise, les quartiers dans les villes importantes sont subdivisés en sous-unités appelées « blocs ». Ceux-ci correspondent à des unités infraterritoriales censées assurer un meilleur maillage municipal du territoire. Chaque bloc a à sa tête un chef de bloc soumis à l'autorité du chef de quartier.
} 
TIC et démocratie locale. Considérations empiriques issues de la pratique du budget participatif dans des communes camerounaises

lorsque nous utilisions les affiches, des communiqués radios ${ }^{4} \ldots$

Les TIC contribuent de ce fait à une forme de restructuration des circuits traditionnels de construction et d'échanges des informations (Lamarche et Villalba, 2005). À la suite d'une communication officielle sur le projet, le maire de Yaoundé 2 rappelait en 2012 :

Cet outil permettra, de renforcer la communication entre les élus locaux que nous sommes et la base pour laquelle nous avons pris l'engagement de servir. Nous pourrons ainsi efficacement associer nos populations [...] dans toutes les phases des projets à réaliser dans nos Communes. Je pense ici prioritairement au choix des projets, à leur suivi, leur évaluation et sur le degré de satisfaction, tout ceci dans le but noble d'assurer l'appropriation de toutes les actions entreprises par les bénéficiaires ${ }^{5}$.

En considérant que l'accès à l'information est l'un des déterminants majeurs de la participation citoyenne aux affaires publiques, les TIC, du moins celles mobilisées dans les expériences discutées ici, ont conséquemment une influence notable sur la participation aux mécanismes décisionnels.

3.1.2. L'implication des citoyens: entre mobilisation, choix des priorités et monitoring

L'implication directe des citoyens aux processus décisionnels est considérée comme étant la clé de voûte d'un cycle complet de participation politique. Elle permet d'aller au-delà de la simple information des citoyens et de s'inscrire dans une approche active de la participation (Verba et Nie, 1987), bien que parfois considérée

\footnotetext{
4 Entretien réalisé le 18 octobre 2015 à Yaoundé, avec le responsable de la mobilisation communautaire dans la mise en œuvre de l'action à ASSOAL. 5 École de la gouvernance du Cameroun (2012), « Le projet TIC pour la gouvernance locale sur les rails, Actualité, <http://gouvernancecameroun.tmpds359.haisoft.net/interne.php?id menu=34\&idsmenu=191\&idarticle $=17>$, dernière consultation le 1 er avril 2017.
} 


\section{Calvin MINFEGUE}

comme étant illusoire. Les TIC sont susceptibles d'apporter des possibilités intéressantes dans ce sens. Le BP, il convient de le rappeler, consiste à associer les citoyens à la prise de décisions relatives aux affectations budgétaires portant sur les investissements publics à réaliser au niveau local. À cet effet, le processus de prise de décisions, dans le modèle développé dans les communes ciblées, s'articule autour de deux grandes séquences s'inscrivant dans une logique progressive. La première consiste à faire participer les citoyens à la définition des projets pouvant être inscrits dans le budget communal. Il s'agit d'une phase de présélection des projets au niveau de chaque unité infraterritoriale, à savoir les quartiers (Douala 3, Yaoundé 2 et Yaoundé 6) et les villages (Edzendouan). On parlera alors, selon les cas, de fora de quartiers ou de villages. La seconde séquence consiste au choix final des projets devant être retenus et exécutés lors du prochain exercice municipal. Ce sont les contraintes budgétaires, autrement dit la disponibilité des fonds et la nature des projets, qui en déterminent le volume/le nombre. Les possibilités offertes par les TIC ont permis, ici, d'agir principalement à deux niveaux: la mobilisation, déterminant majeur de la participation à la prise de décisions et le suivi de la réalisation des projets.

Les usages des TIC dans la mobilisation des citoyens s'avèrent déterminants lors de la tenue des fora de quartiers ou de villages à partir du moment où ils permettent d'élargir l'assise sociale de la participation et de donner plus de légitimité aux choix opérés à cette étape de présélection. Ce sont les effets des TIC sur les niveaux de mobilisation des citoyens lors de ces fora (en vue de la présélection des projets potentiellement inscriptibles sur l'agenda municipal lors de l'exercice suivant) qui sont ici considérés comme étant des éléments capitaux du processus décisionnel associé au BP. À Yaoundé 2 et Edzendouan, on se rend compte qu'avec les TIC, les niveaux de participation des citoyens aux fora ont connu une relative évolution.

À Yaoundé 2 (Tableau 1), on note une augmentation significative de la participation citoyenne entre $2010(0,26 \%)$ et $2011(0,48 \%)$, à la suite d'une intensification de l'action communicative et d'une forte implication des organisations communautaires dans l'animation du 
TIC et démocratie locale. Considérations empiriques issues de la pratique du budget participatif dans des communes camerounaises

processus. La progression entre 2011 et 2015, à la suite de l'introduction des TIC s'est ensuite avérée beaucoup plus faible $(0,48 \%$ à $0,52 \%)$. Il semblerait donc que la hausse constatée en 2015 ait aussi bénéficié de la politique antérieure favorable au développement de la participation.

Tableau 1. Évolution de l'affluence aux fora de quartiers en 2010, 2011 et 2015 à Yaoundé 2

\begin{tabular}{c|c|c|c|} 
& $\mathbf{2 0 1 0}$ & $\mathbf{2 0 1 1}$ & \multicolumn{2}{c}{$\mathbf{2 0 1 5}$ (TIC) } \\
\cline { 2 - 4 } $\begin{array}{c}\text { Effectif de la } \\
\text { participation aux fora } \\
\text { Pourcentage de la } \\
\text { population }\end{array}$ & 704 & 1331 & 1640 \\
\cline { 2 - 4 } & $0,26 \%$ & $0,48 \%$ & $0,52 \%$ \\
\hline
\end{tabular}

Source : données de terrain et compilation données ASSOAL.

À Edzendouan (Tableau 2), la proportion de la population participant aux fora est passée de 4,28\% en 2007 à 10,94\% en 2015. Cette forte hausse doit néanmoins être relativisée, car elle apparaît davantage associée à une intense activité communicative qu'à un effet spécifique des TIC. En effet, selon nos informations, cette commune connaît des difficultés d'accès à l'électricité, pourtant nécessaire en vue de recharger les téléphones portables. A cet égard, les TIC (spécifiquement les téléphones portables utiles à la réception des informations) apparaissent forcément jouer un rôle secondaire.

Tableau 2. Évolution de l'affluence aux fora de villages en 2004, 2007 et 2015 à Edzendouan

\begin{tabular}{c|c|c|c|}
\multicolumn{1}{c}{$\mathbf{2 0 0 4}$} & $\mathbf{2 0 0 7}$ & $\mathbf{2 0 1 5}$ (TIC) \\
\cline { 2 - 4 } $\begin{array}{c}\text { Effectif de la } \\
\text { participation aux fora } \\
\begin{array}{c}\text { Pourcentage de la } \\
\text { population }\end{array}\end{array}$ & - & 201 & 736 \\
\cline { 2 - 4 } & - & $4,28 \%$ & $10,94 \%$ \\
\hline
\end{tabular}

Source : données de terrain et compilation données ASSOAL. 


\section{Calvin MINFEGUE}

On peut donc conclure à une incidence progressive, mais relative des TIC, tant dans les cas de Yaoundé 2 que d'Edzendouan. Néanmoins, le rôle des TIC devrait être apprécié sur une durée plus longue dans le cadre d'analyses plus fines.

Les TIC sont par ailleurs mobilisées dans le suivi de la réalisation des projets issus du BP. Deux actions peuvent être distinguées ici. La première mobilise la plateforme SMS. Les citoyens ayant leurs numéros dans la base de données téléphoniques reçoivent des messages indiquant les projets issus du processus du BP, tout en spécifiant lesquels seront réalisés dans leurs quartiers. La seconde action exploite un centre d'appel "citoyen» mis en place par l'ASSOAL et fonctionnel depuis la fin d'année 2013. Il recense les appels provenant des citoyens désireux de fournir des informations sur l'exécution des projets, initialement identifiés dans le cadre du BP. Ces informations sont centralisées, analysées et transmises aux autorités compétentes. Ces dernières entreprennent par la suite des démarches de vérification et, quand cela est nécessaire, engagent des actions correctives. Cela a été le cas à Yaoundé 2. De nombreuses plaintes (près de 2000 à la fin d'année 2015) ont été reçues par le centre d'appel. Ces plaintes portaient sur le caractère défectueux de certaines infrastructures d'approvisionnement en eau et sur le mauvais état de certaines voies. Le transfert de ces informations à la municipalité a conduit les autorités publiques à entreprendre ou à susciter des travaux de réparation, dans le cas d'infrastructures relevant de leur compétence et issues du processus de BP. Deux situations de ce type ont pu être, par exemple, relevées : la réfection des points d'approvisionnement en eau dans des quartiers tels que Messa-carrière ou Briqueterie et la réhabilitation d'une voie d'accès au quartier Nkomkana à Yaoundé 2.

Même si le suivi semblait initialement orienté vers les municipalités mettant en œuvre le BP, le centre d'appel a reçu également de nombreuses plaintes ciblant des services publics, comme l'approvisionnement en eau potable et l'électricité. Il a ainsi reçu, entre 2013 et 2015, plus d'une centaine de plaintes destinées aux sociétés publiques concernées, à savoir la Camerounaise des eaux (CDE) pour l'approvisionnement en eau et l'entreprise The Energy of Cameroon (ENEO) pour l'approvisionnement en électricité. Des 
TIC et démocratie locale. Considérations empiriques issues de la pratique du budget participatif dans des communes camerounaises

alertes sur des pannes de transformateur électrique à Yaoundé ou la qualité de l'eau à Douala ont très souvent été reçues et transmises aux sociétés concernées. Néanmoins, ces plaintes n'ont pas toujours été suivies de mesures correctives immédiates. L'hypothèse peut donc être faite, ici, que ces dispositifs de suivi facilités par les TIC devraient aller de pair avec une augmentation des capacités structurelles des acteurs en position d'apporter des réponses et à agir de manière efficace face aux demandes des usagers, des citoyens. La mobilisation des TIC dans de telles démarches doit dès lors être associée à des structures suffisamment organisées et performantes.

Un troisième niveau de mobilisation des TIC existe, à savoir leur emploi pour effectuer le choix des priorités à partir des projets présélectionnés. Cette décision finale se déroule dans le cadre d'une assemblée où tous les habitants de la commune votent les projets sur la base d'une liste préexaminée. Ces projets sont analysés en lien avec leur faisabilité et leur cohérence par rapport aux domaines de compétences municipales. Ce préexamen s'effectue au cours du forum des délégués élus lors des fora de quartiers ou de villages. En fonction du montant du budget, les projets qui seront inscrits dans le plan de campagne de l'exercice suivant sont choisis en fonction du nombre de suffrages recueillis. Les projets sont donc choisis selon la quantité des voix recueillies jusqu'à épuisement du budget à affecter. Les TIC interviennent ici dans l'élargissement du bassin de votants potentiels. Elles permettent ainsi une implication plus large au processus décisionnel.

Dans les processus traditionnels, le vote s'effectue lors des assemblées communales se tenant dans un espace pouvant contenir le maximum de citoyens (place publique). Chaque citoyen a la possibilité de choisir le projet qu'il estime prioritaire. Partant du constat fait par les acteurs, des niveaux d'affluence très faibles lors des assemblées précédentes, une option supplémentaire a été explorée : la possibilité de voter par SMS. À Yaoundé 6 (Tableau 3), commune ayant expérimenté ce vote en 2012, on a observé une hausse de l'ordre de $268 \%$ du nombre de votants, comparativement à 2011. 


\section{Calvin MINFEGUE}

\section{Tableau 3. Évolution de la participation populaire dans les processus de BP à Yaoundé 6 entre 2010 et 2012*}

\begin{tabular}{|c|c|c|c|}
\multicolumn{2}{c}{} & \multicolumn{2}{c|}{$\begin{array}{c}2012 \\
\text { [avec l'usage } \\
\text { des SMS) }\end{array}$} \\
\hline $\begin{array}{c}\text { Nombre de personnes touchées } \\
\text { directement par les informations } \\
\text { relatives au processus }\end{array}$ & 1500 & 25000 & 45000 \\
\hline $\begin{array}{c}\text { Participation aux fora de quartiers } \\
\text { (nombre de personnes) }\end{array}$ & 481 & 301 & 982 \\
\hline $\begin{array}{c}\text { Participation à l'assemblée } \\
\text { communale (nombre de } \\
\text { personnes) }\end{array}$ & 231 & 204 & 810 \\
\hline $\begin{array}{c}\text { Nombre de personnes ayant } \\
\text { participé au vote final des priorités }\end{array}$ & 231 & 204 & 751 \\
\hline
\end{tabular}

*Les chiffres pour l'année 2012 sont un cumul des effets liés à la fois au processus traditionnel et à l'usage des TIC.

Source : Compilation des données ASSOAL/CAY 6.

Une précaution s'impose cependant dans l'interprétation des données disponibles. Il y a une différence fondamentale entre le processus traditionnel de vote, qui est encadré par des interactions directes, physiques permettant justement une meilleure expression des volontés et des opinions citoyennes au-delà des choix à effectuer. Par contre, le vote par SMS n'autorise pas nécessairement cette dynamique interactive dont il ne faut pas négliger l'influence dans les choix définitifs des citoyens. Le risque existe donc de rationaliser de façon (trop) importante un processus censé favoriser la participation populaire.

L'accès à l'information et le renforcement de l'implication aux processus décisionnels apparaissent comme des finalités associées aux usages manifestes des TIC dans le processus. Toutefois, les géographies particulières des territoires et les logiques des acteurs s'y déployant révèlent des usages détournés. 
TIC et démocratie locale. Considérations empiriques issues de la pratique du budget participatif dans des communes camerounaises

\subsection{Des usages politiques détournés dans les sphères locales}

Au-delà des usages manifestes orientés vers l'amélioration de la participation des citoyens au BP, des usages diffus, parfois inattendus, détournés, peuvent être identifiés. Ils correspondent, ici, à des usages non identifiés par les acteurs comme fondamentaux ou inhérents aux TIC dans le processus. Il s'agit en fait d'usages secondaires, qui se manifestent parfois insidieusement et qui sont liés aux logiques spécifiques des acteurs impliqués dans la mise en œuvre du BP. À cet égard, deux formes ont pu être identifiées: l'emploi des TIC comme canal d'élargissement de l'audience politique au niveau local et leur mobilisation comme outil d'impulsion et de renforcement des dynamiques réticulaires.

3.2.1. Un canal d'élargissement de l'audience politique au niveau local

Les potentialités que les TIC génèrent en ce qui a trait à la mobilisation d'un grand nombre de citoyens avec la possibilité d'un contact personnalisé avec eux ne sont pas sans intérêt pour les acteurs politiques locaux, en l'occurrence les élus. C'est le cas à Yaoundé 2. Sur ce territoire, les acteurs politiques considèrent que les effets des TIC peuvent se déployer sur deux plans: une mobilisation croissante des citoyens et une politisation « subtile » du bilan des usages des TIC.

Dans le contexte de la commune de Yaoundé 2, les possibilités accrues de mobilisation des citoyens ont été exploitées par l'équipe municipale en 2013. Ainsi, informer les citoyens en vue de les inciter à participer aux fora de quartiers et aux travaux collectifs d'assainissement public (nettoyage des voies d'accès et des édifices publics, curage des caniveaux notamment) a souvent constitué pour les élus un moment de diffusion de messages ayant une connotation clairement politique. L'ampleur de telles communications a même été renforcée en période préélectorale, par exemple entre janvier et juin 2013. Prétextant leur engagement à participer aux journées de travail citoyen, le maire de la commune a incité ses concitoyens à « toujours faire confiance à l'équipe municipale ${ }^{6}$ ». Il y a là un usage

${ }^{6}$ Discours du maire lors d'une journée de propreté en mars 2013. 


\section{Calvin MINFEGUE}

détourné des opportunités offertes par les TIC en vue de les orienter vers des finalités s'écartant clairement des activités consacrées au BP. Cet usage imprévu procède, dans le cas d'espèce, d'un double séquençage: une explicitation de la finalité attribuée à l'outil (mobilisé pour le BP) et une exploitation de l'espace, du cadre offert à des fins communicatives tout à fait décalées par rapport aux objectifs initiaux.

Les activités associées au BP ont toujours été inscrites dans le bilan de l'exécutif municipal de Yaoundé 2 depuis 2008. L'expérience est présentée comme une pratique novatrice des élus locaux en vue de renforcer la participation citoyenne aux affaires publiques locales. L'inclusion des TIC à ce processus a davantage renforcé cette capitalisation politique. Les TIC sont présentées par les acteurs comme un moyen supplémentaire permettant d'étendre les possibilités d'inclusion des citoyens. Dans ce cas, l'accent est également mis sur la dimension "moderne » d'une telle initiative, qui reflète elle-même la logique "modernisante » de l'action municipale. Cette modernité, dans l'acception des acteurs municipaux, se situe à deux niveaux. D'une part, elle prend pied dans l'inclusion d'un volet TIC au BP; d'autre part, elle se manifeste dans la capacité de la municipalité à s'ouvrir à de "nouveaux » outils. Cette référence à la modernité est très souvent apparue dans les discours électoraux du maire de Yaoundé 2 lors de la campagne de 2013. Un leader communautaire, membre d'un comité de quartier, affirmait à cet effet :

La mobilisation des TIC est devenue un fonds de commerce politique pour l'équipe municipale. Elle s'en sert pour marquer notamment la présence du maire auprès des populations et faire surtout remarquer ses différentes actions. Mais c'est de bonne guerre ${ }^{7} \ldots$

Il est donc possible de constater que, même si les TIC sont mobilisées pour favoriser la participation des citoyens, les élus exploitent toujours ce déploiement à leurs fins. Ils ouvrent l'espace de participation, mais gardent néanmoins le contrôle. Dans de telles

${ }^{7}$ Entretien réalisé le 22 janvier 2014 à Yaoundé. 
TIC et démocratie locale. Considérations empiriques issues de la pratique du budget participatif dans des communes camerounaises

circonstances, l'action semblerait s'inscrire davantage dans un modèle d'e-politics (concernant les logiques et les positionnements politiques d'acteurs très politisés) que d'e-democracy.

3.2.2. L'impulsion et le renforcement des dynamiques réticulaires

À Douala 3 et Edzendouan, la mobilisation a permis, de façon diffuse, l'impulsion et le renforcement, selon les cas, des dynamiques réticulaires. Celles-ci font référence aux mobilisations, aux engagements et aux pratiques des acteurs associatifs. À Douala 3, les associations communautaires de base étaient peu impliquées dans les champs d'intervention relevant de la gouvernance locale. Leurs actions portaient sur des enjeux économiques tels que l'appui à la production et le développement des activités génératrices de revenus. L'expérimentation du BP, dès 2013, a permis aux structures associatives d'investir le champ de la gouvernance locale en mettant l'accent sur la mobilisation communautaire. Ce changement de cap s'est agencé autour de deux infrastructures «TIC» : la plateforme Web-SMS et le centre d'appel.

À propos du premier dispositif, la campagne de sensibilisation relative à la constitution d'une base de données téléphoniques (utile à la diffusion des messages) a été en partie animée par des associations de quartiers à l'instar de «Solidarité PK 9 » ou «Famille du Nde ». Concernant le second dispositif, la même approche informative a permis aux organisations communautaires de quartiers de sensibiliser les citoyens aux possibilités de recours au centre d'appel afin de signaler d'éventuels problèmes dans la conduite des projets. Ces associations ont également porté l'organisation des fora de quartiers en mettant à disposition des animateurs, fins connaisseurs des territoires. Dans les quartiers de cette commune, 54 animateurs provenant d'une vingtaine d'associations ont ainsi été mobilisés. L'usage des TIC, notamment l'envoi des SMS aux animateurs, a assuré leur mobilisation et leur information sur l'organisation des fora. Cette dynamique a permis à ces entités associatives de s'inscrire dans une collaboration formalisée avec la municipalité de Douala 3.

Dans le sillage de ces changements, certaines associations, en capitalisant sur les possibilités induites par le centre d'appel, se sont 
orientées vers le contrôle citoyen des interventions publiques au niveau local. Le contrôle citoyen est compris, ici, comme un suivi alternatif des politiques publiques par les citoyens organisés en structures communautaires. C'est le cas de "Famille du Nde», association qui s'est spécialisée dans le suivi des projets issus du BP et ceux relevant des interventions publiques. Cette association documente leur exécution et en partage les résultats sur un réseau socionumérique, en l'occurrence Facebook, tout en transmettant ces informations à la municipalité. Il apparaît donc que les TIC, dans le contexte de la commune de Douala 3, ont permis aux associations communautaires d'investir de nouveaux champs d'intervention. Cela leur permet d'être présentes dans l'espace public et de favoriser l'émergence d'un processus démocratique plus continu que celui fondé sur les traditionnelles élections.

À Edzendouan, les associations locales qui se sont impliquées dans ces activités étaient déjà engagées en 2004, aux premières heures de l'expérience du BP dans cette commune. Avec l'arrêt de l'expérience entre 2007 et 2012, elles ont limité leurs activités à des actions sporadiques de sensibilisation communautaire autour des programmes de santé ou d'organisation d'activités lucratives au sein de structures telles que le "Service Environnemental, Santé et Développement». Le redémarrage du processus en 2013 leur a donné une nouvelle impulsion. Il est ici intéressant de constater que l'utilisation des TIC sert de prétexte à l'impulsion de certaines activités, telles que des campagnes d'informations autour du centre d'appel et de la constitution de la base de données. Elle donne ainsi à des entités associatives en dormance ou au fonctionnement intermittent des opportunités de réaffirmer leur présence dans l'espace public.

\section{TIC et démocratie locale au Cameroun : une association positive et viable ?}

L'e-démocratie n'altérerait pas radicalement, dans divers contextes, le modèle démocratique dominant, comme l'ont relevé 
TIC et démocratie locale. Considérations empiriques issues de la pratique du budget participatif dans des communes camerounaises

certains auteurs (dont Barber, 1984). Toutefois son apport, ou du moins l'analyse que l'on peut en faire, est ambivalente.

\subsection{Des effets des TIC sur la dynamique démocratique à l'épreuve du local...}

D'un côté, l'e-démocratie trouverait dans le «local » camerounais un terreau fertile de transformation des modalités de participation des citoyens, en diversifiant leurs modes d'implication. Les possibilités qu'elles induisent permettraient de dépasser les « limites techniques » du débat et de la participation classiques (TournadrePlancq, 2010). Il y a là, peut-être, l'idée d'une mutation subtile des modalités d'expression démocratiques. Les possibilités sont perceptibles sur divers plans : informatif et formatif du citoyen, si l'on se situe dans le sillage des réflexions de Dahl (1998); participatif, enjeu prisé par les tenants d'une démocratie plus directe ; et, enfin, décisionnel, enjeu ultime. Les déficits de transparence souvent évoqués, qui favoriseraient des pratiques clientélistes ou corruptives (Chabal et Dalloz, 1999; De Sardan, 2008 ; Médard, 2000), tant au Cameroun que plus largement sur le continent africain, pourraient aussi être minimisés par ces outils. Les TIC permettraient aux sociétés du continent de s'arrimer à " l'impératif de transparence et de redevabilité (accountability) qui gagne les démocraties libérales depuis quelques années et dessine de nouveaux contours à la légitimité des gouvernants » (Tournadre-Plancq, 2010, p. 80, emphase de l'auteur). La diffusion des informations portant sur les budgets, telle qu'elle est présentée dans les cas abordés ci-dessus, en constitue une bonne illustration.

Les TIC, par leur mobilisation sociopolitique, permettraient de renforcer substantiellement l'action de la société civile. Elles favoriseraient la création d'une chaîne vertueuse de pratiques démocratiques, plus constantes, donnant de la voix à la société civile et renforçant progressivement les mécanismes de redevabilité. La société civile, justement, devrait jouer un rôle important de facilitateur dans l'accès des citoyens ordinaires à l'information, d'une part (Gigler, Bailur et Anand, 2014). D'autre part, elle devrait intervenir dans la construction d'un dialogue fécond entre acteurs 


\section{Calvin MINFEGUE}

(élus, citoyens). C'est dans ce sens qu'il faudrait analyser l'action des organisations sociales.

Pour finir, il semblerait que les TIC soient virtuellement capables de redéfinir, voire de requalifier, la trame même de l'espace public habermassien, lequel espace se situerait hypothétiquement entre un espace public aux matérialités empiriquement observables et un espace public virtuel façonné, alimenté par les TIC et aux matérialités spécifiques. Cet espace public virtuel relève de l'élargissement des "habiletés civiques» et du développement des ressources dont disposent les citoyens pour mieux l'investir (Bastien, 2013). Il traduit donc une "réappropriation » de l'espace public traditionnel par des acteurs aux profils variés (Tréguer, 2013). Il en ressortirait donc un espace hybride qui, en réalité, aurait comme marqueurs différentiels majeurs les possibilités de saisissement, les modalités de participation dont disposeraient désormais les citoyens. Les TIC «impulsent donc une citoyenneté active et renouvellent ses formes précédentes » (Pailliart, 1995, p. 71).

\section{2.... à des effets contreproductifs contextuellement motivés}

D'un autre côté, il semblerait que l'e-démocratie ne redéfinisse pas fondamentalement les rapports de pouvoirs et les logiques politiciennes à l'œuvre dans les systèmes représentatifs. En effet, les TIC font, à différents niveaux, l'objet d'une méfiance de la part des acteurs politiques qui les déploient en vue d'en contrôler les effets. Ces résultats sont exploités afin de renforcer, dans les faits, les traits des systèmes politiques et aussi, donc, la " centralité de l'élu » (Jouve, 2005). Les marges de manœuvre accordées aux citoyens n'empêchent pas en réalité l'élu de garder le contrôle : "Doter les citoyens ne dépossède en aucun cas le Prince» (Caillosse, 1999, p. 78). Certes, les données empiriques présentées ne font pas forcément état de manière rigoureuse du fameux «paradoxe de l'intermédiaire» qui voit l'acteur politique à la fois comme le promoteur des modèles d'e-démocratie (puisqu'il en favorise souvent l'avènement) et comme le principal opposant d'une plus grande implication citoyenne (Mahrer et Krimmer, 2005). On n'en est toutefois pas éloigné. 
TIC et démocratie locale. Considérations empiriques issues de la pratique du budget participatif dans des communes camerounaises

Les contextes sociaux présentent aussi, dans leur dynamique, des facteurs limitatifs. En effet, la capacité des citoyens à utiliser les TIC (digital literacy) reste un facteur déterminant (Gigler, 2011). Elle conditionne la portée des effets qu'on escompte d'une mobilisation des TIC. De très grandes inégalités subsistent entre citoyens (alphabétisés/analphabètes, urbains/ruraux, riches/pauvres, etc.) en matière d'accès aux techniques. Ces inégalités existent également par rapport à la disponibilité ou pas de dispositifs spécifiques répondant aux contraintes du contexte. Les inégalités, très souvent décrites comme une contrainte du jeu démocratique, semblent donc être perpétuées par l'e-démocratie. À titre d'exemple, Edzendouan voit la portée d'une mobilisation intense des TIC réduite du fait des difficultés d'approvisionnement en électricité et de sa couverture aléatoire par les réseaux téléphoniques.

Par ailleurs, il est important de noter que les performances de l'edémocratie dépendent fortement de celles initiales du système qu'elle veut améliorer. De ce fait, comme l'a précisé Liden (2012), elle devient le «miroir » du système politique. Elle ne sera réellement «démocratique » que si le contexte initial l'est. Cela suppose donc de distinguer clairement les processus politiques facilités par les TIC $(e-$ politics) des processus démocratiques facilités par les TIC (edemocracy). Dans le cas du présent texte, sommes-nous situé dans l'epolitics ou dans l'e-démocracy? La question demeure.

Les TIC ne semblent donc pas reconfigurer systématiquement, en contexte camerounais comme ailleurs, le modèle démocratique. Les TIC, non loin d'être des catalyseurs effectifs d'une revigoration de la pratique démocratique, restent des catalyseurs potentiels (Gigler, Bailur et Anad, 2014). Elles apportent néanmoins des changements qualitatifs dans la façon dont les processus politiques propres au système au sein duquel elles se déploient sont configurés et actés. L'e-démocratie serait donc, au Cameroun, une réalité qui se cherche.

\subsection{Désinvestir et réinvestir autrement les études sur les TIC}

De toute évidence, il importe de (re)penser la problématique «TIC et démocratie ». Cela nécessite de construire ou de constituer un corpus de connaissances sur la mobilisation des TIC dans les dynamiques politiques. Il semble y avoir, en effet, un vide dans la 


\section{Calvin MINFEGUE}

recherche sur les usages des TIC dans les contextes africains (Benchenna, 2012) du fait de contraintes liées à la maturation de cet objet, mais, également, du fait d'une certaine immobilité dans les contenus consacrés à la notion d'usage. Le développement des recherches aux croisements de celles portant sur les TIC et celles consacrées aux processus politiques dans une perspective assez décentrée, comme la sociologie des usages, serait intéressant et même souhaitable. Il permettrait d'explorer les (autres) voies, pour aborder différemment les TIC dans les processus sociaux, par exemple, comme le suggère Avgerou (2011), par les approches structuralistes (des pratiques), les approches sociotechniques et les approches institutionnalistes. À celles-ci, on pourrait ajouter l'approche par les capacités, mise en exergue par les travaux de Gigler, Bailur et Anand (2014).

Dans les cas camerounais explorés ici, les TIC se présentent davantage comme une option d'optimisation des structures, des actions politiques classiques. En effet, réduire les TIC dans de telles initiatives à une fonction de complément optimisant conduit à mésestimer leurs capacités à redéfinir les structures et les actions auxquelles elles sont associées. Il est sans doute opportun d'envisager que l'adjonction des TIC dans les processus politiques aboutisse à des modèles hybrides. Peut-être n'en sommes-nous pas encore là. La question de la pérennité de tels dispositifs dans un environnement de fragilité financière (vu le coût de l'infrastructure en TIC) interpelle parallèlement la nécessaire volonté politique.

Que retenir de cette réflexion sur les TIC dans les processus politiques au niveau local en contexte camerounais ? D'abord, dans le cas des expériences mobilisées, l'influence de facteurs extérieurs remet en question les modalités de leur usage. Ensuite, les TIC font l'objet, dans ces processus politiques, d'une mobilisation ambivalente s'organisant autour d'usages parfois détournés. Enfin, avec du recul, il semble que l'e-démocratie permette des avancées d'ordre qualitatif, notamment dans le renouvellement des formes d'engagement citoyen. Toutefois, de nombreuses contraintes repérables dans le modèle démocratique classique demeurent, à l'instar des tensions entre logiques politiciennes et objectifs politiques, voire démocratiques. Il y a là nécessité d'observer ces formes nouvelles de 
TIC et démocratie locale. Considérations empiriques issues de la pratique du budget participatif dans des communes camerounaises

pratiques politiques sur un temps long. Quoi qu'il en soit, la réflexion se poursuit...

\section{Références bibliographiques}

ART, 2016, Observatoire annuel 2014 du marché des communications électroniques, Yaoundé, Agence de Régulation des télécommunications.

AVGEROU C., 2011, "The Developmental Role of ICT », Communication présentée à la London School of Economics and Political Science, Information Systems and Innovation Group, Londres, <https://www.dmst.aueb.gr/Documents/Conferences/Ten_ye ars_DET_files/Chrisanthi\%20Avgerou\%20$\% 20 A U E B \% 20$ anniversary.pdf>, dernière consultation le 1 er avril 2017.

BARBER B., 1997, Démocratie forte, Paris, Desclée de Brouwer.

BLONDIAUX L., G. MARCOU, F. RANGEON, P. DEYON, A. MABILEAU et J. CAILLOSSE (dir.), 1999, La démocratie locale. Représentation, participation et espace public, Paris, Presses universitaires de France.

CAILLOSSE J., 1999, "Éléments pour un bilan juridique de la démocratie locale en France», dans L. BLONDIAUX, G. MARCOU, F. RANGEON, P. DEYON, A. MABILEAU et J. CAILLOSSE (dir.), 1999, La démocratie locale. Représentation, participation et espace public, Paris, Presses universitaires de France, pp. 63-78.

CHABAL P. et J.-P. DALOZ, 1999, Africa Works: Disorder as Oolitical Instrument, Oxford, James Currey.

CUSTER S., N. NOVIN et E. PALUMBO, 2011, «Social Media Enabled Beneficiary Feedback Loops: Mitigating Risk in Technological Innovation », World Bank Institute, Washington DC, Innovation Practice. 


\section{Calvin MINFEGUE}

DE SARDAN J-P. O., 2008, «À la recherche des normes pratiques de la gouvernance réelle en Afrique», Afrique, pouvoir et politique, $\mathrm{n}^{\circ} 5$, $<\mathrm{http}$ ///www.institutionsafrica.org/filestream/20090109-discussion-paper-5-larecherche-des-norms-pratiques-de-la-gouvernance-r-elle-enafrique-jean-pierre-olivier-de-sardan-d-c-2008>, dernière consultation le 1er avril 2017.

GEORGE É., 2014, «Quelles perspectives critiques pour aborder les TIC ?», tic\&société, vol. 8, $\quad \mathrm{n}^{\circ} 1-2, \quad<\mathrm{http}: / /$ ticetsociete.revues.org/1365>, dernière consultation le 1 er avril 2017.

GIGLER B.-S., 2011, " "Informational Capabilities": The Missing Link for the Impact of ICT on Development», E-Transform Knowledge Platform Working Paper 1, World Bank, Washington, DC.

GIGLER B.-S., S. BAILUR et N. ANAND, (dir.), 2014, Closing the Feedback Loop: Can Technology Bridge the Accountability Gap?, Washington, Directions in Development, World Bank.

GOUO L. B., 2008, La réforme administrative dans les services publics en Afrique, Paris, L'Harmattan.

HEATER D., 2004, A Brief History of Citizenship, New York, New York University Press.

HELLER P. et V. RAO (dir.), 2015, Deliberation and Development: Rethinking the Role of Voice and Collective Action in Unequal Societies, Washington, World Bank.

INS, MINEPAT, MINSANTE, 2012, Enquête démographique et de santé et à indicateurs multiples (EDS-MICS), Yaoundé, Ministère de l'Économie, de la Planification et de l'Aménagement du Territoire.

LAMARCHE T. et B. VILLALBA, 2005, «La domestication d'internet dans le jeu politique », Terminal, $\mathrm{n}^{\circ} 92$, <http://www.revueterminal.org/www/articles/92/introInternetPol.html>, dernière consultation le 1er avril 2017. 
TIC et démocratie locale. Considérations empiriques issues de la pratique du budget participatif dans des communes camerounaises

LAUDON K. 1977, Communication Technology and Democratic Participation, Londres, Sage Publications.

LIDEN G, 2012, "Is e-Democracy More Than Democratic? An Examination of the Implementation of Socially Sustainable Values in e-Democratic Processes ", Electronic Journal of eGovernment, vol. 10, $\mathrm{n}^{\circ}$ 1, pp. 84-94.

MADON S., 2000, «The Internet and Socio-Economic Development: Exploring the Interaction», Information Technology and Peoples, vol. 13, n² 2, pp. 85-01.

MAHRER H. et R. KRIMMER, 2005, "The 'Middleman Paradox' in Enhancement of e-Democracy », Information Systems Journal, $\mathrm{n}^{\circ} 15$, pp. 27-42.

MEDARD J.-F., 2000, "Clientélisme politique et corruption », TiersMonde, vol. 41, nº 161 pp. 75-87.

PAILLIART I., 1995, "Démocratie locale et nouvelles techniques d'information et de communication », Pouvoirs, n 73, pp. 6978.

PEIXOTO T., 2008, e-Participatory Budgeting: e-Democracy From Theory to Success?, Zurich, E-democracy Centre, e-working papers.

PNUD, 2000, Rapport du PNUD sur la pauvreté. Vaincre la pauvreté humaine, New York, PNUD.

RODOTÀ S., 1999, La démocratie électronique. De nouveaux concepts et expériences politiques, Rennes, Apogée.

ROUSSEAU D. (dir.), 1995, La démocratie continue, Paris, LGDJBruylant.

SAVAH J. et A. GALABOV (dir.), 2012, Participations et citoyennetés depuis le printemps arabe, Paris, L'Harmattan.

TOURNADRE-PLANCQ J., 2010, « La démocratie électronique : quelles attentes? », Cahiers français, $\mathrm{n}^{\circ}$ 356, p 77-81.

VERBA S. et N.H. NIE, 1987, Participation in America: Political Democracy and Social Equality, Chicago, University of Chicago Press. 


\section{Calvin MINFEGUE}

WEBER B., 2010, «ICT4GOV Mobile Phone Technology to Empower Citizens to Support Better Governance», communication, Washington, World Bank Institute.

WORLD BANK, 2011, I Paid a Bribe: Learning from ICT Facilitated Access to Information Innovations in South Asia, One World Foundation, Delhi.

WORLD BANK INSTITUTE, 2012, «Information and Communication Technology for Governance (ICT4GOV) Program. Case study contributing to the Study on Strengthening Inclusive Stakeholder Ownership through Capacity Development», World Bank capacity Development and results.

ZAKHOUR L., 2011, «Printemps arabe : de l'imaginaire au réel. Les moyens d'information et de communication font la révolution », Hermès, n 61 , pp. 212-218. 